\title{
Measurement of Arbitrary Strain Profiles of Fiber Bragg Gratings in Fabry-Perot-like Transmission Spectra Using a Real-Coded Genetic Algorithm
}

\author{
Hsu-Chih Cheng, Chih-Ta Yen ${ }^{1, *}$ and Yong-Jing Xiao ${ }^{2}$ \\ Department of Electro-Optical Engineering, National Formosa University, \\ No. 64, Wunhua Rd., Huwei Township, Yunlin County 632, Taiwan \\ 'Department of Electrical Engineering, National Formosa University, \\ No. 64, Wunhua Rd., Huwei Township, Yunlin County 632, Taiwan \\ ${ }^{2}$ Institute of Electro-Optic Science and Engineering, Department of Physics, \\ National Cheng Kung University, Tainan City 701, Taiwan
}

(Received January 23, 2014; accepted March 13, 2014)

Key words: chirped fiber Bragg grating, distributed strain sensing, Fabry-Perot-like, real-coded genetic algorithm

In this paper, we propose a method of measuring arbitrary strain distribution using real-coded genetic algorithm optimization processes to analyze the reflection-intensity spectra of Fabry-Perot-like fiber Bragg gratings (FBGs). The reflected spectra produce Fabry-Perot-like effects, and the grating is subjected to the strain field. Because of the Fabry-Perot-like effect, strain profiles produce different reflection-intensity spectra. Thus, the arbitrary strain distributions along the embedded length of FBGs can be immediately obtained from the Fabry-Perot-like reflective spectra. The correctness of the proposed methods was demonstrated numerically by reconstructing the strain distributive profile in the FBGs, to which several different nonuniform strains were applied (linearly negative gradient, linearly positive gradient, and discontinuity strains). The proposed methods were demonstrated to enable accurate strain reconstruction with no restrictions on the applied strain profile.

\section{Introduction}

Fiber Bragg gratings (FBGs) have become the most crucial sensing components for measuring distributed strain variations in smart structures. Huang et al. proposed three different distributed grating interrogation systems based on the measured reflective intensity spectrum of the grating and/or the measured phase spectrum..$^{(1-5)}$ However, the reflective intensity-spectrum-based approach and the phase-spectrum-based approach are only suited to continually increasing or decreasing distributed strain profiles.

"Corresponding author: e-mail: chihtayen@gmail.com 
Reconstruction of the arbitrary strain distribution within fiber gratings using timefrequency signal analysis was proposed by Azana et al.;(6,7) this method allows accurate strain reconstruction with no restrictions on the applied strain profile, but requires both intensity and phase spectra to be measured. At present, simultaneous measurement of intensity and phase spectra is complicated and requires expensive equipment. Casagrande et al. ${ }^{(8)}$ described a genetic algorithm approach to solve an inverse problem in optics, by which the characteristics of a uniform FBG are determined according to its reflected spectrum. The validity of the proposed method was demonstrated using a Bragg sensor to measure nonlinear strain acting on a uniaxial aluminum test specimen. Unfortunately, the system is only able to measure strain profiles monotonically (continually increasing or decreasing). Cheng and Lo presented a novel method of recovering the distribution of the period of an FBG using an arbitrary distributed strain sensing application. In their approach, a uniform FBG and a chirped FBG were used to extract two strain-induced reflection intensity spectra. A genetic algorithm was then applied to these spectra to reconstruct the arbitrary strain distribution along the two parallel FBGs. Cheng and Lo proposed a novel method of extracting multiple parameters of arbitrary FBGs using a genetic algorithm and two thermally modulated reflection intensity spectra; they demonstrated the ability of the proposed method to determine multiple parameters of uniform and chirped FBGs. ${ }^{(9,10)}$ Recently, Rodriguez-Coboa et al. have proposed a new processing scheme based on a comparison metric, geometrical processing, and a blind optimization technique, particle swarm optimization (PSO), to resolve axial perturbation applied to FBGs. ${ }^{(11)}$ The simulation results were consistent with those of experiments. In this paper, the strain-distribution sensing method based only on the Fabry-Perot-like reflective spectra of FBGs is presented.

\section{Theory}

\subsection{Transfer matrix}

The spectral profile of an FBG was simulated using the piecewise-uniform approach (transfer matrix approach). ${ }^{(12)}$ In this approach to modeling an FBG structure, $2 \times 2$ matrices for each uniform section of the grating were identified, and all of them were multiplied together to obtain a single $2 \times 2$ matrix that describes the entire grating. The compound grating structure was divided into $M$ uniform $k$. Therefore, to determine the spectral profile of an FBG, the boundary condition matrix components were first determined, and $F_{k}^{+}$and $B_{k}^{+}$were the field amplitudes obtained after traversing the sections $F_{0}^{+}=F^{+}(L / 2)=1, B_{0}^{+}=B^{+}(L / 2)=0$; these were used to calculate the final matrix components $F_{M}^{+}=F^{+}(-L / 2)=0$ and $B_{M}^{+}=B^{+}(-L / 2)=0$. The propagation through each of the uniform sections $k$ was described using a matrix $\left[T_{k}\right]$, which is defined as

$$
\left[\begin{array}{c}
F_{k}^{+} \\
B_{k}^{+}
\end{array}\right]=\left[T_{k}\right]\left[\begin{array}{c}
F_{k-1}^{+} \\
B_{k-1}^{+}
\end{array}\right],
$$


where the matrix $\left[T_{k}\right]$ is given by

$$
\left[T_{k}\right]=\left[\begin{array}{cc}
\cosh (\Omega d z)-i \frac{\zeta^{+}}{\Omega} \sinh (\Omega d z) & -i \frac{\kappa}{\Omega} \sinh (\Omega d z) \\
i \frac{\kappa}{\Omega} \sinh (\Omega d z) & \cosh (\Omega d z)+i \frac{\zeta^{\dagger}}{\Omega} \sinh (\Omega d z)
\end{array}\right]
$$

where $d z$ is the length of the $k$ th uniform section, $\zeta^{+}$and $\kappa$ are the local coupling coefficients for the $k$ th section, and

$$
\Omega=\sqrt{\kappa^{2}-\zeta^{+2}}
$$

The total grating structure is expressed as

$$
\left[\begin{array}{c}
F_{M}^{+} \\
B_{M}^{+}
\end{array}\right]=\prod_{j=1}^{M}\left[T_{j}\right]\left[\begin{array}{l}
F_{0}^{+} \\
B_{0}^{+}
\end{array}\right]=T\left[\begin{array}{l}
F_{0}^{+} \\
B_{0}^{+}
\end{array}\right]=\left[\begin{array}{ll}
T_{(11)} & T_{(12)} \\
T_{(21)} & T_{(22)}
\end{array}\right]\left[\begin{array}{c}
F_{0}^{+} \\
B_{0}^{+}
\end{array}\right]
$$

Thus, the coefficients of reflection $R$ are given by

$$
R(\lambda)=\left\|\frac{T_{(21)}}{T_{(11)}}\right\|^{2} .
$$

Consequently, the theoretical reflectivity spectrum is obtained by numerically calculating $R(\lambda)$ at each wavelength within the spectral range.

\subsection{Bragg-grating-based Fabry-Perot cavity}

The transfer matrix approach permits the calculation of the reflection of a Bragggrating-based Fabry-Perot cavity. Briefly, the matrix $T_{\mathrm{FP}}$ characterizing a Bragg-gratingbased Fabry-Perot cavity is expressed as in ref. 13,

$$
T_{\mathrm{FP}}=\prod_{j=1}^{M}\left[T_{\text {grating } 1, j}\right] \cdot T_{\mathrm{ps}} \cdot \prod_{j=1}^{M}\left[T_{\text {grating } 2, j}\right] .
$$

where the matrices $\prod_{j=1}^{M}\left[T_{\text {grating1 }, j}\right]$ and $\prod_{j=1}^{M}\left[T_{\text {grating } 2, j}\right]$ are the transfer matrices of gratings 1 and 2 , respectively. The matrix $T_{\mathrm{FP}}$ is the phase-shift term (two gratings separated by a homogeneous length $e$ ) and is defined as

$$
T_{\mathrm{ps}}=\left[\begin{array}{cc}
\exp \left(\frac{-i 2 \pi n_{\mathrm{eff} e}}{\lambda}\right) & 0 \\
0 & \exp \left(\frac{i 2 \pi n_{\mathrm{eff} e}}{\lambda}\right)
\end{array}\right] .
$$




\subsection{Real-coded genetic algorithm}

In the proposed sensing system, strain profiles produce different reflection-intensity spectra as a result of the Fabry-Perot-like effect. Therefore, the arbitrary strain distribution can be inversely reconstructed using the real-coded genetic algorithm optimization process, as shown in Fig. 1(a). In Fig. 1, the strain-optic effect is applied in the genetic algorithm model and the strain profile $\varepsilon_{f}(z)$ to yield a sample string of real values.

The error function (fitness function) is a measurement of the distance between the calculated reflective spectrum $R_{\text {calc }, j}$ and the target reflective spectrum $R_{\text {target } j \text {. }}$.

The error function determines how the genetic algorithm weighs different parts of the spectrum. The error function is similar to that in ref. 14 and is expressed in eq. (8).

$$
\text { Error_Function }=\sum_{N}\left(R_{\mathrm{target}, j}-R_{\mathrm{calc}, j}\right)^{2} .
$$

In the genetic algorithm crossover process, two parent species were randomly chosen, their bit data were exchanged, and two other species were generated. By accumulating exceptional bit information as this process was repeated, more satisfactory generations were produced. The probability in the crossover process was controlled by crossover probability. The real-value crossover process was executed as in ref. 14.

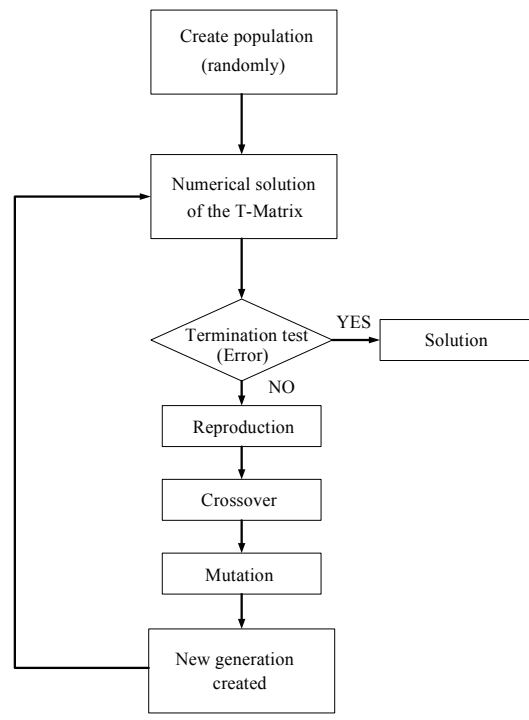

(a)

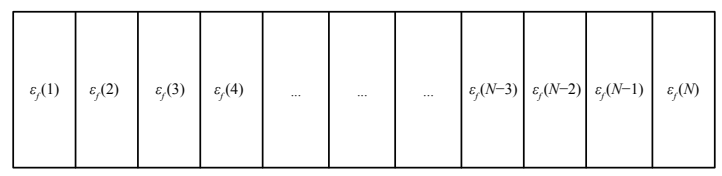

(b)

Fig. 1. (a) Flow chart of genetic algorithm. (b) Sample strings of real values. 


\section{Linearly Chirped FBG Fabry-Perot Sensor}

\subsection{System description}

In the proposed sensing system, as shown in Fig. 2(a), two identical linearly chirped FBGs constitute a series and are placed closely together. Both of these linearly chirped FBGs form a Fabry-Perot sensor of broadband spectra embedded in a stressed structure; the two gratings are subjected to the same strain field. The original reflective spectra of the two gratings from 1548 to $1557 \mathrm{~nm}$ are shown in Fig. 2(b). Figure 2(a) shows a schematic of the linearly chirped grating Fabry-Perot cavity sensor system. A wavelength-tunable laser was used as a coherent light source; the laser was coupled through a $3 \mathrm{~dB}$ coupler, transmitted to the Fabry-Perot cavity sensor system, and reflected to a power meter. The proposed sensor comprises two colocated linearly chirped FBGs with opposite chirped orientations; thus, this linearly chirped FBG cavity configuration formed multiple spectral Fabry-Perot cavities with various cavity lengths, ${ }^{(15,16)}$ and thus, can be used as an arbitrary strain sensor. If the chirped orientations of the two linearly chirped FBGs were identical to each other, the two strain fields (uniform and linearly positive gradient strain fields), which were specifically designed to differ, would have produced the same reflective spectra. Therefore, only the linearly chirped grating cavity, with the opposite chirped orientation, was used to complete the measurement of the arbitrary strain profiles.

In arbitrary strain sensing using this type of linearly chirped FBG cavity as a sensor head, the strain on the sensor head induces a shift in the Fabry-Perot resonance wavelength. Thus, strains produce different reflective intensity spectra. This method allows the arbitrary strain distribution to be immediately recovered, enabling the reflective intensity spectra to be analyzed using the real-coded genetic algorithm optimization processes, as shown in Fig. 1(a).

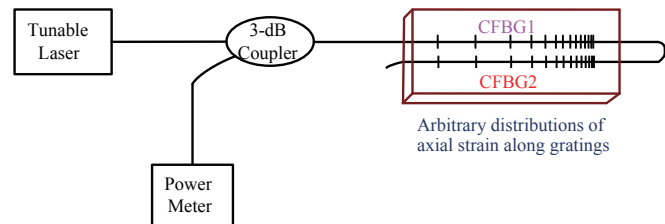

(a)

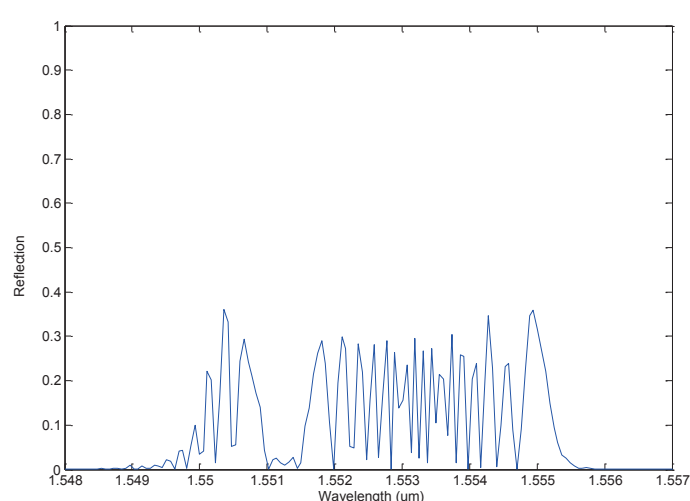

(b)

Fig. 2. (Color online) (a) Proposed sensing system; (b) original chirped FBG spectra of two gratings without applied strain. 


\subsection{Results of numerical simulation}

The validity of this proposed sensing system was demonstrated by applying the two different strain profiles to the Fabry-Perot sensor head, and using the real-coded genetic algorithm optimization processes to determine the optimal solution.

In this simulation, the fiber axis was assumed parallel to the $\mathrm{z}$ direction, and the strain was applied to the z-axis. The effective index of the fiber core was selected as $n_{\text {eff }}=1.45775$. The grating length was $1 \mathrm{~cm}$. The index of modulation depth was $\Delta n=1.25 \times 10^{-4}$, the chirped value was $5.5 \mathrm{~nm} / \mathrm{cm}$, and the start period was $0.533 \mu \mathrm{m}$. The two gratings were separated in an optical fiber at a distance of $20 \mathrm{~cm}$.

Figure 3 shows the sensor in a linearly positive gradient strain and nonmonotonic

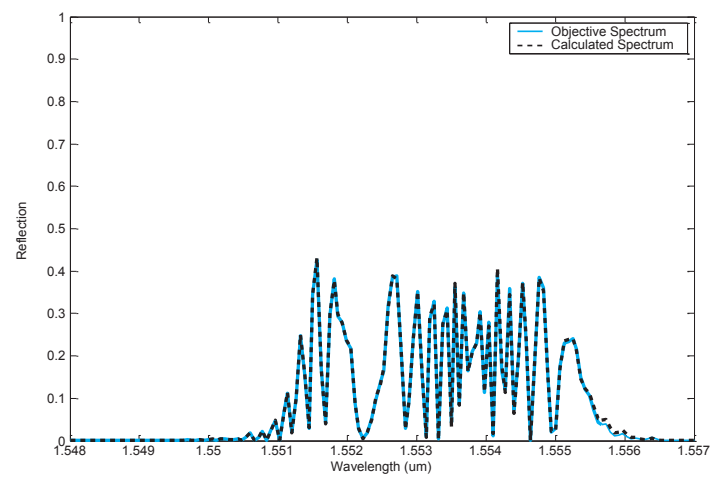

(a)

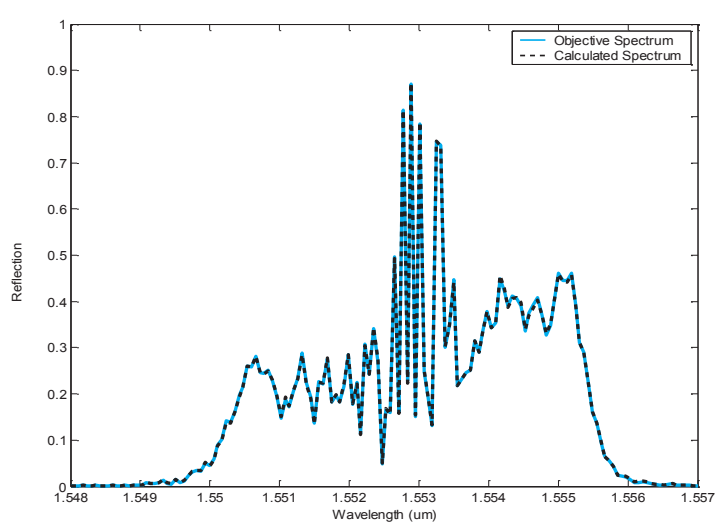

(c)

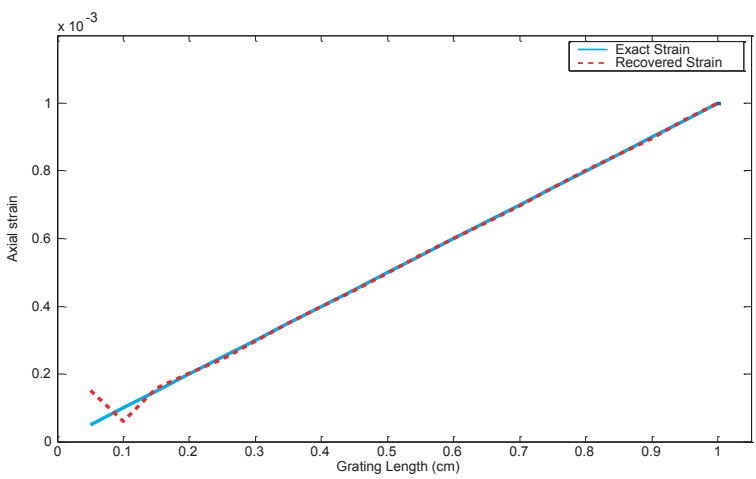

(b)

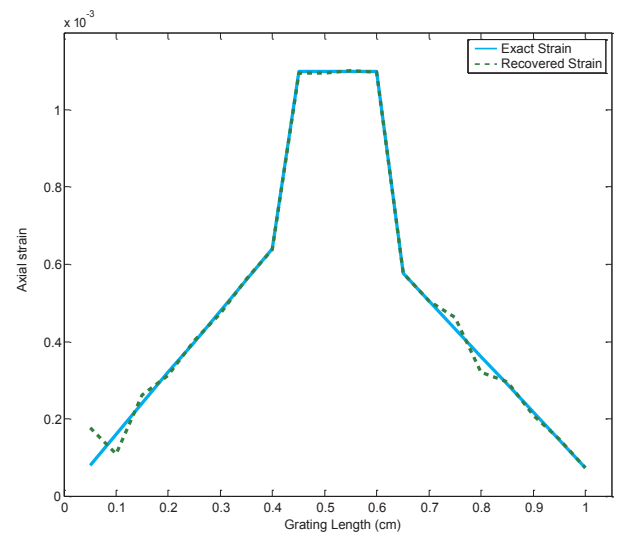

(d)

Fig. 3. (Color online) (a) Reflective spectrum under linearly positive gradient strain (solid line) and optimal reflective spectrum with genetic algorithm (dashed line); (b) linearly positive gradient ideal strain (solid line) and recovered strain with genetic algorithm (dashed line); (c) reflective spectrum under nonmonotonic distribution strain (solid line) and optimal reflective spectrum with genetic algorithm (dashed line); (d) nonmonotonic ideal distribution strain (solid line) and recovered strain with genetic algorithm (dashed line). 
strain fields. As shown in Fig. 3(a), the optimal reflective spectrum (dashed curve) and objective spectrum (solid curve) are consistent with each other. The strain recovered using the genetic algorithm (dashed line) is close to the linearly positive gradient ideal strain (solid line), as shown in Fig. 3(b). After 10000 iterations of the real-coded genetic algorithm, the error value from eq. (8) was 0.0203 .

After 10000 iterations of the real-coded genetic algorithm, the error value from eq. (8) was 0.0397 . As shown in Fig. 3(d), the discontinuity strain distribution was recovered over the entire grating length with satisfactory accuracy. The simulation results demonstrated that the sensor had no limitations on the applied strain profile, and could be used as an arbitrary strain sensor.

\section{Conclusions}

In this paper, the method of conducting arbitrary strain distribution sensing using a real-coded genetic algorithm to analyze the reflective intensity spectra of Fabry-Perotlike FBGs is presented. In general, grating-based Fabry-Perot cavity sensors can only measure the point strain; measuring the true strain distribution along the grating length has been impossible. Nevertheless, in this study, use of the grating-based Fabry-Perot cavity sensor was proposed. The system exhibited superior performance in measuring the strain distribution, and was able to recover the arbitrary strain profile along the grating length.

Applying certain techniques, it is difficult to use a single FBG (a uniform FBG or a linearly chirped FBG) to recover the arbitrary strain distribution based on the reflective intensity spectrum alone, because two specifically different strain fields exist in the same reflective intensity spectrum. The systems proposed in this study are simple and ideal for a number of smart structure applications, and permit accurate strain reconstruction with no restrictions on the applied strain profile.

\section{Acknowledgements}

This work was supported in part by the National Science Council under Grant No. NSC-102-2622-E-150-016-CC3.

\section{References}

1 S. Huang, M. M. Ohn, M. LeBlanc, R. Lee and R. M. Measures: Proc. 1994 Conf. Distrib. Multiplexed Fiber Optic Sensors IV 2294 (1994) 81.

2 S. Huang, M. LeBlanc, M. M. Ohn and R. M. Measures: Appl. Opt. 34 (1995) 5003.

3 S. Huang, M. M. Ohn and R. M. Measures: Appl. Opt. 35 (1996) 1135.

4 R. M. Measure, M. M. Ohn, S. Y. Houang, J. Bigue and N. Y. Fan: Smart Mater. Struct. 7 (1998) 237.

5 S. Huang, M. M. Ohn, M. LeBlanc and R. M. Measures: Smart Mater. Struct. 7 (1998) 248.

6 J. Azana and M. A. Muriel: Opt. Lett. 25 (2000) 698.

7 J. Azana, M. A. Muriel, L. R. Chen and P. W. E. Smith: J. Lightwave Technol. 19 (2001) 646.

8 F. Casagrande, P. Crespi, A. M. Grassi, A. Lulli, R. P. Kenny and M. P. Whelan: Appl. Opt. 41 (2002) 5238 . 
9 H. C. Cheng and Y. L. Lo: Opt. Commun. 239 (2004) 323.

10 H. C. Cheng and Y. L. Lo: J. Lightwave Technol. 23 (2005) 2158.

11 L. Rodriguez-Cobo, A. Cobo and J. M. Lopez-Higuera: Opt. Lett. 38 (2013) 2327.

12 A. Othonos and K. Kalli: Fiber Bragg Gratings: Fundamentals and Applications in Telecommunications and Sensing (Artech House, Boston, 1999) pp. 197-200.

13 S. Legoubin, M. Douay, P. Bernage and P. Niay: Opt. Soc. Am. A 12 (1995) 1687.

14 Z. Michalewicz: Genetic Algorithms + Data structure = Evolution Programs, 3rd ed. (SpringerVerlag, New York, 1996).

15 P. Torres and L. C. G. Valente: Opt. Commun. 208 (2002) 285.

16 K. P. Koo, M. LeBlanc, T. E. Tsai and S. T. Vohra: IEEE Photonics Technol. Lett. 10 (1998) 1006. 